\title{
EEG-based Computer Control Interface for Brain-Machine Interaction
}

\author{
http://dx.doi.org/10.3991/ijoe.v11i6.5119 \\ J. Katona and A. Kovari \\ University of Dunaújváros, Dunaújváros, Hungary
}

\begin{abstract}
Recently more and more research methods are available to observe brain activity; for instance, Functional Magnetic Resonance Imaging (fMRI), Positron Emission Tomography (PET), Transcranial Magnetic Stimulation (TMS), Near Infrared Spectroscopy (NIRS), Electroencephalograph (EEG) or Magnetoencephalography (MEG), which provide new research opportunities for several applications. For example, control methods based on the evaluation of measurable signals of human brain activity. In the past few years, more mobile EEG (electroencephalogram) based brain activity biosensor and signal processing devices have become available not only for medical examinations, but also to be used in different scopes; for instance, in control applications. These methods provide completely new possibilities in human-machine interactions by digital signal processing of brain signals. In this study, the program model, the establishment, the implementation and the test results of the quantitative EEG-based computer control interface, protocol and digital signal processing application are demonstrated. The user-friendly visualization of the evaluated brain wave signals is implemented in visual $C \#$ object-oriented language. This EEG-based control unit and interface provides an adequate basis for further research in different fields of brain-machine control methods regarding the examination of possible machine control applications.
\end{abstract}

\section{INTRODUCTION}

To observe brain activity, a number of devices primarily designed for medical applications can be used. Much research is in progress to observe brain signals in different stages, which can be used in the development of braincontrolled devices among others. To observe brain activity, the following devices are mostly used:

\section{A. Functional Magnetic Resonance Imaging (fMRI)}

From the brain mapping technologies listed in the abstract, the most modern is the so-called functional magnetic resonance imaging examination, which can also be used to observe brain activity. This device detects the change of oxygen supply of the blood, which is also called the hemodynamic answer of the brain [1-3].

Nerve activities occurring in the brain can be observed during the bloodstream. When one brain field becomes more active, it takes more oxygen delivered by the haemoglobin in the erythrocyte and it causes larger bloodstream, which occurs after brain activity 1-5 seconds later. The bloodstream reaches its peak in 4-5 seconds, then it falls down to its basic level $[12,13]$.
By using the fMRI activity map, brain parts participating in certain mental processes as well as the long-term memory or learning processes can be examined [1-4].

\section{B. Positron Emission Tomography (PET)}

The positron emission tomography (PET) and fMRI technology are non-invasive techniques to view brain activity. The PET device combined with the Computed [Axial] Tomography (CT) is one of the most modern imaging diagnostic technologies. The basis of the operation of the positron emission tomography is ensured by the molecules signed with isotopes beaming positrons, which makes it possible to visualize the biochemical reactions of the human body $[5,6]$.

In contrast with the fMRI technology, PET technology uses molecules containing radioactive isotopes that dissolve due to positron emission during its operation and has to be taken into the human system. This means that the examination involves radiotherapy [6].

During the examination, images showing primarily the slices perpendicular to the longitudinal axis of the body can be taken. After the process, three dimensional images can be reconstructed in any direction from the slices [7].

\section{Transcranial Magnetic Stimulation (TMS)}

The transcranial magnetic stimulation is an electrophysiological process that ensures the possibility to explore bonecovered or deep-laying nerve structures in a non-invasive way $[8,9]$.

During the TMS process, a copper ring is placed above the skull, the alternation of the magnetic field created by electric current in the ring can penetrate the skull and this creates current in the involved area [9]. The magnetic impulse generated by TMS can usually alternate between 1.5 and 4 Tesla, which causes electric current in the nerve tissues and can cause depolarization of the neural membrane at adequate intensity [10].

\section{Functional near-infrared spectroscopy (fNIRS)}

fNIR is a non-invasive imaging method to measure the chromophore concentration by near infrared (NIR) light attenuation, temporal or phasic changes. Using fNIR, brain activity can be measured through hemodynamic responses of neurons. fNIR relies on the principle of neuro-vascular coupling, the Hemodynamic or BOLD (BloodOxygenation-Level-Dependent) response. This principle is similar to fMRI techniques, so fNIR and fMRI are sensitive to similar physiologic changes and highly correlated results in cognitive tasks [18]. 
fNIR is much cheaper than fMRI and it is a portable device, but cannot be used to measure cortical activity more than $4 \mathrm{~cm}$ deep.

\section{E. Electroencephalograph (EEG)}

To examine continuous nerve activity of the brain, electrophysiological methods can be used, primarily electroencephalograph (EEG) or rarely magnetoencephalography (MEG). Electroencephalograph is an electrophysiological measuring device and it can record the electric activity of neurons in real time. The signal that can be led by EEG, is called the electroencephalogram, which is a complex, multi-component, periodic curve.

The electroencephalogram can be recorded by two methods: in an invasive and a non-invasive way. In case of the first method, some micron diameter micro electrodes are placed in the brain tissue by drilling a hole through the skull. This method is primarily used in animal experiments, while in case of humans, the non-invasive method is used, by placing low resistance metal macro electrodes on the scalp.

During EEG examinations, the potential difference is always observed between two electrodes. These measurements can be both bipolar, when the curve recorded on two different points of the skull is evaluated by correlation to each other, and unipolar, when potential alternation is compared to a curve recorded by an indifferent or inactive electrode. Leads that do not convey neural activity (a point far from the skull, e. g. the earlobe) are called inactive, reference electrode, while those that detect voltage change as a result of neural activity, are called active electrodes [19-21].

The EEG device measures bioelectric brain signals by electrodes placed on the scalp and visualizes them graphically. The electrodes detect the EEG signals that are generated by voltage pulses of a certain field of the brain and the device examines their alternation and spectrum [14, 22]. The recorded EEG signals above the examined brain field, can provide significant information regarding the activity of a certain brain field [19, 22].

\section{F. Comparison and application possibilities of brain activity measuring devices}

The fMRI and PET-CT are very expensive and not portable equipment, though they are high resolution brain mapping devices. Therefore, they are used in medical applications only. Furthermore, they have inadequate time resolution. TMS also has some disadvantages; for example, the details of its effect mechanism is not known [11], and it can have unpleasant side effects $[9,10]$.

The time resolution of fNIRS and EEG-based devices are better; in addition, the newly developed devices are portable. As a result of recent developments, EEG-based mobile devices have become relatively cheap and available; therefore, apart from medical applications, they can be used in other research as well. In the last few years, many research projects have started in the field of human brain controlled machines that apply EEG-based devices.

For instance, EEG technology is used in case of ASIMO (Advanced Step in Innovative Mobility), a device that is a robot similar to a humanoid boy and can be controlled by brain waves [23].

Furthermore, at the German Technical University of Berlin, an EEG signal processing-based system have been developed that can detect the braking intent of the driver 130 milliseconds earlier compared to the braking by pressing the brake pedal. Due to this time difference, the stopping distance of a $100 \mathrm{~km} / \mathrm{h}$ fast vehicle could be reduced even by 3.7 meters [24].

At the University of Minnesota, a brain-wave controlled helicopter that is able to pass through an obstacle course have been developed. The EEG signals were measured by a cap with 64 electrodes and the helicopter was controlled the processing of the brain waves that occurred by the imagination of different movements (left or right fist grips) [25].

In this study, I present a universal EEG-based computer control interface to implement brain-machine interactions using an open source control protocol.

\section{HUMAN BRAIN ACTIVITY}

The flow of electrons and ions in the cell induces electric current, which generate voltage on the resistance of this area [1]. Synaptic transmission and the contact places between the cells, through which the stimulus covers from cell to cell, is called synapse (Fig. 1). It is mainly evoked by the cell body of a neuron (axon) and the nerve-ending of another neuron (dendrite). The stimulus transmitter is called presynaptic and the carrier is postsynaptic. During the transmission, the two neurons do not abut totally, the stimulus is transmitted by the so-called neuron transmitters. Once the stimulus is transmitted, the presynaptic nerve excretes chemicals that stimulate the postsynaptic nerve $[19,21]$.

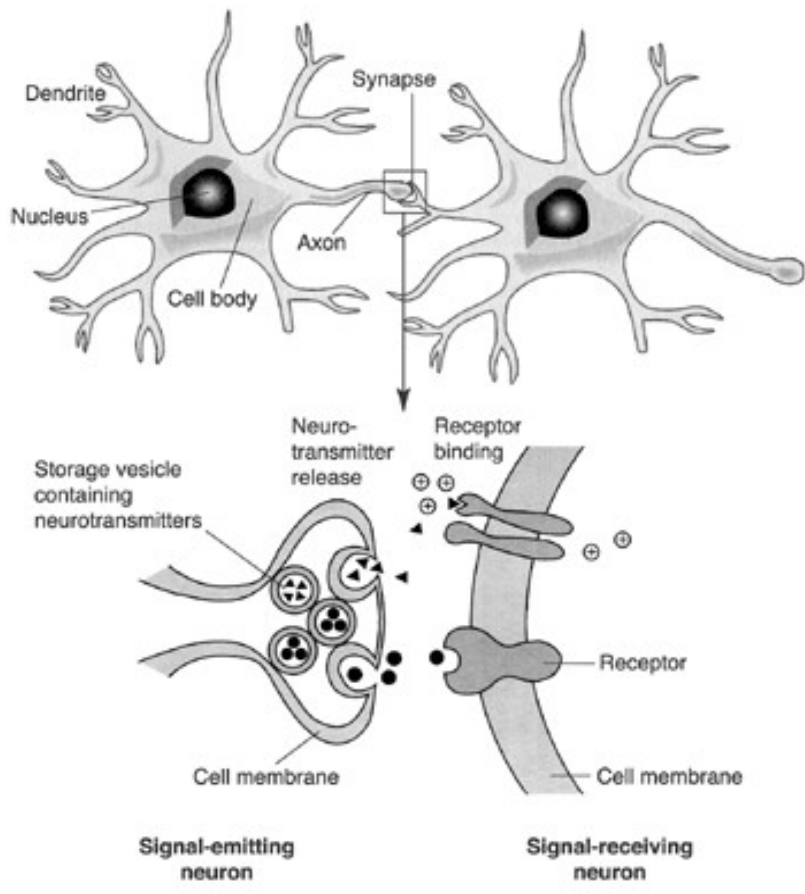

Figure 1. Synapse, the moment of stimulus transmission.

Regarding human brain, it can be claimed that its specific functions during operation are well known. The interpretation of brain waves would not be possible without understanding the operation of the brain, through which the brain waves produced by brain activity are aimed to retrieve and investigate. Such activity can be observed by examining the electrical and magnetic phenomenon of 
neural functions by the widespread method called electrophysiology.

Brain activity, brain waves and the neural functions of the brain are mostly observed by electroencephalogram (EEG). The processing of EEG signals is usually executed by quantitative EEG (QEEG), by which the frequency spectrum of EEG signals can be investigated [15, 22].

The EEG signal is a complex multi-component periodical wave, which is rich in relatively larger amplitude (8-12 $\mathrm{Hz}$ ) waves in calm state. The exact source of the EEG signals has not been discovered in details so far. Probably these signals occur as a consequence of aggregated electric activity of several, thousands of neurons located on the surface of the cerebral cortex. Bioelectric signals generated in the brain, or the electric activity of neurons induce some electromagnetic fields. This means that voltage alternations occur by cortex activity.

\section{BRAIN WAVES}

Brain activity is shown in the spectrum of measurable EEG signals. When our brain becomes ,excited" its „talk” becomes faster, just like in case of humans. What our brain wants to ,tell" us, can mainly be seen in low frequency waves, between approximately 1 and 12-14 Hz. The occurrence of frequency components is determined by alertness level: in case of increased alertness, generally, high frequency components are experienced, whereas in deep sleep stages delta waves occur.

The detected brain signals given by the EEG device are mostly investigated by Fourier analysis, which separates the signals into frequency components resulting the spectrum of the detected brain wave [22]. Each brain wave frequency component has a specific functional significance as shown in Table I.

TABLE I.

FREQUENCY COMPONENTS OF BRAIN WAVES

\begin{tabular}{|c|c|c|}
\hline $\begin{array}{c}\text { Brain wave } \\
\text { Type }\end{array}$ & $\begin{array}{c}\text { Frequency } \\
\text { range }\end{array}$ & $\begin{array}{c}\text { Mental states and condi- } \\
\text { tions }\end{array}$ \\
\hline Delta & $0.1 \mathrm{~Hz}$ to $4 \mathrm{~Hz}$ & $\begin{array}{c}\text { Deep, non-REM sleep, } \\
\text { unconscious }\end{array}$ \\
\hline Theta & $4 \mathrm{~Hz}$ to $8 \mathrm{~Hz}$ & $\begin{array}{c}\text { Intuitive, creative, recall, } \\
\text { fantasy, imaginary, dream }\end{array}$ \\
\hline Alpha & $8 \mathrm{~Hz}$ to $13 \mathrm{~Hz}$ & $\begin{array}{c}\text { Relaxed, but not drowsy, } \\
\text { tranquil, conscious }\end{array}$ \\
\hline Low Beta & $13 \mathrm{~Hz}$ to $16 \mathrm{~Hz}$ & $\begin{array}{c}\text { Formerly SMR, relaxed } \\
\text { yet focused, integrated }\end{array}$ \\
\hline Midrange Beta & $16 \mathrm{~Hz}$ to $20 \mathrm{~Hz}$ & $\begin{array}{c}\text { Thinking, aware of self } \\
\text { and surroundings }\end{array}$ \\
\hline High Beta & $20 \mathrm{~Hz}$ to $30 \mathrm{~Hz}$ & Alertness, agitation \\
\hline Gamma & $40 \mathrm{~Hz}$ to $80 \mathrm{~Hz}$ & $\begin{array}{c}\text { Motor functions, higher } \\
\text { mental activity }\end{array}$ \\
\hline
\end{tabular}

IV. THE MOBILE EEG DEVICE

The MindFlex is an innovative control device based on ThinkGear technology developed by NeuroSky and it was introduced in 2009. The device controls the levitating of a ball based on the evaluation of brain wave types that occur during the processing of electric biosignals of the brain received by the EEG headset. The measured real-time brain wave intensity is evaluated by the algorithm running in the micro controller in the EEG headset or in the control computer similarly to other real-time computer systems used in engineering practice $[5,6]$.
The headset has three sensors; one of them measures the frontal lobe and the other two should be placed on the lower parts of the ear (reference points). Thanks to ThinkGear technology, the digital signal processor in the EEG headset can determine the magnitude of concentration. [16]

The EEG headset forwards the processed signals to the controlled unit (Fig. 2) in a wireless but non-standard WiFi connection [3]. As a result of this, wired serial connection is used to evaluate the EEG data. On this serial line, the read of the processed data sent by the EEG headset was done by an USB-UART decoder [4].

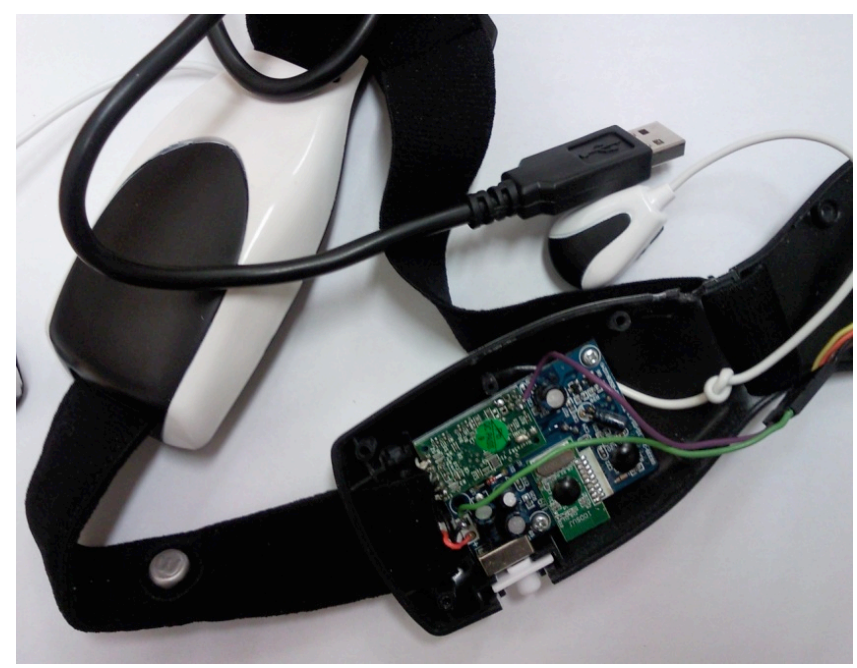

Figure 2. The MindFlex EEG headset.

\section{ATTENTION LEVEL AND BRAINWAVES}

The connection between attention and brain waves was already observed in the 1970s and an EEG-based attention analyzer was patented (US Patent Number: 3877466, 1975). The method of measuring attention is based on the examination of the spectrum strength determined by the FFT algorithm of brain waves (Cooley, 1965). As a result of the observations, they found that the strength of the alpha brain waves in high attention state is rather low. However, the amplitude of alpha brain waves in low attention state is large. The observed difference between the changes of the brain waves is the base of the patented attention analyzer. The parameters of brain waves can be determined by digital signal processing algorithms, its spectrum can be calculated with discrete Fourier transformation (DTF) algorithm and the results can be evaluated based on the received brain waves strengths. With this method, the strength of brain waves from the spectrum and the level of attention can be defined.

$$
X_{k}=\sum_{n=0}^{N-1} X_{n} \cdot e^{-i \cdot 2 \pi k \cdot \frac{n}{N}} \quad k=0, \ldots, N-1
$$

\section{VERY SIMPLE CONTROL PROTOCOL (VSCP)}

VSCP is a free to use package with open software and firmware tools, highly scalable application level protocol for machine to machine automation tasks. This protocol does not assume anything about the lower level system and can be used for TCP/IP, Ethernet, CAN, USB, RS-232 
etc. The complete VSCP has two levels of communication, Level I is designed to CAN but Level II version of the protocol is intended for Ethernet or Wi-Fi. All share the same common message fields and framework. The main advantages of this protocol are its autonomous, distributed device functionality, uniform device configuration, uniform device discovery and identification. [28] In a VSCP network, a common physical layer connects the individual nodes/devices to form the control network. This network is a distributed system with all nodes working autonomously.

VSCP and friends is a collected software package, a complete solution for measurement and control. VSCP uses a well specified message format and the protocol supports global unique identifiers for nodes. It automatically assigns a unique ID to a newly installed node and inform other nodes and possible hosts that a new node is available and ready. It has a register model to give a common interface to node configuration and a model for controlling node functionality. [28]

VSCP is an event-based system. Nodes generate events and nodes react on events. Normally events are not addressed but instead are broadcast on the bus. Each node on the network will receive the event and will decide if this event needs to be handled or not. The example shows a button being pressed Node 1 sending an event message onto the bus informing all others and Node 3 receives the message and decides this button should trigger Lamp 2 to turn on (Fig. 3).

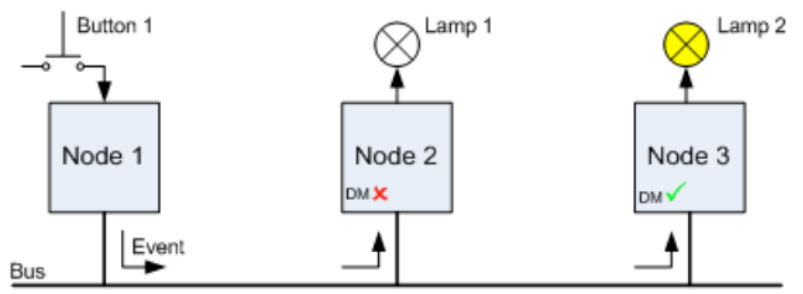

Figure 3. Communication between Nodes

All nodes can optionally implement a decision matrix. This matrix is used to define one or a group of events that should trigger a predefined functionality at the module. Events are defined into classes and types; for example, measurements, information and control. If an event is received, it should be handled by the decision matrix (DM) of the node. All events have an originating address, a GUID - consisting of 16 bytes but a shorter, typically, one byte nickname ID is used on most systems - for the node they are sent from. It is always possible to deduce the full GUID from the nickname. Events are divided into groups. First, there is Level I and Level II events. Level I events are limited to a maximum of eight bytes of data, while Level II events can have up to 488 bytes of data. [29]

A software package called VSCP \& Friends is available to support VSCP users and it contains the VSCP daemon. The server makes it possible to control several VSCP segments over the Internet. The server has secure Internet interface and makes it possible to add drivers for segments of nodes or special equipment. A driver can communicate with the server using the CANAL interface for a Level I driver and the TCP/IP interface for a Level II driver. VSCP Works is a client application which can be used to send/receive VSCP events to/from every segment/device that export a CANAL interface and remote VSCP daemon.
VSCP is an easy and cost effective way to build systems with distributed intelligence. The protocol is open source, so it is free for anyone to use and modify to their own needs.

\section{THE IMPLEMENTATION OF THE BRAIN VISUALIZATION AND EVALUATION SOFTWARE}

The magnitude of brain wave signal is determined with an EEG (electroencephalogram) measuring and processing unit, produced by NeuroSky. The read, the conversion and the various visualization of the measured and processed signals are implemented in the developed software.

A Windows Forms Application has been developed to evaluate and visualize the brain wave data of the MindFlex EEG headset, described in the previous chapter [17]. This program can run on a PC and was implemented in C\#. The following requirements have been considered regarding the visualization and evaluation software:

- it should be able to receive data sent through serial communication by the EEG headset;

- it can check if any data transfer errors have emerged during transmission;

- it stores data in adequate format and structure for further procession;

- it illustrates processed data in a column chart for evaluation;

- it can execute data evaluation faster than the data sending speed of the headset;

- in case of the PC software, the investigation of signals and display of the measured and processed data alternation has also been achieved to be able to observe changes in the brain wave signals.

For the software development Microsoft Visual Studio was applied. This development environment supports modern object-oriented programming on Windows operating system.

The developed program can be divided into four main functions: the EEG device data communication, the data processing and analysis, the visualization; furthermore, the control communication with nodes. The EEG device data communication function reads, converts the data received from the headset through serial connection. The data processing and analysis function realize the FFT spectrum analysis, the visualization part displays the spectrum by a column chart and the time function of the brain signal by a line chart. The control communication part realizes the control interactions with other nodes using VSCP protocol. The user interface can be seen in Figure 4.

The source code contains three classes: one of them is the so-called BrainWaveReaderForm, which deals with events and derives from the Form class which derives from the source of all classes named Object. BrainWaveReaderForm is responsible for data processing and control communication between other nodes. The second class is called the FormGraphics class and is responsible for data visualization and is in connection with the BrainWaveReaderForm class. The third class is the SerialPortManag$e r$, which manages the EEG serial port communication. In Figure 5, you can see the flowchart illustrating the main functions of the program. 
PAPER

EEG-BASED COMPUTER CONTROL INTERFACE FOR BRAIN-MACHINE INTERACTION

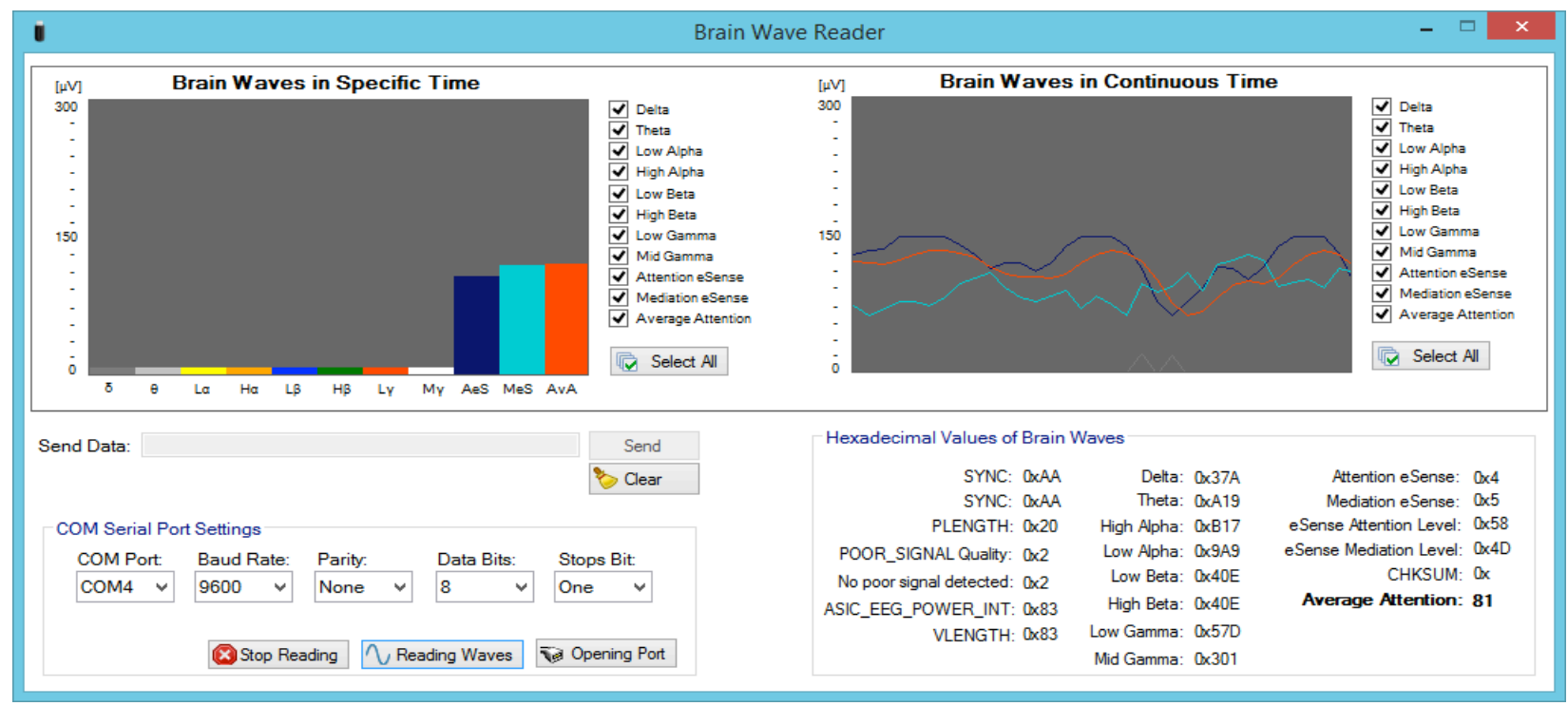

Figure 4. User Interface of BCI data process and visualization software

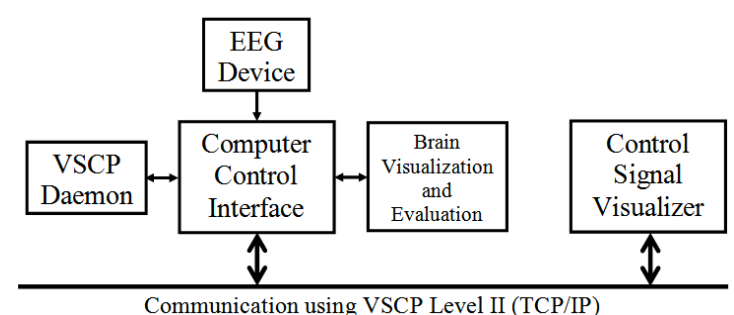

Figure 5. Test environment for control interface

\section{EVALUATION}

The Computer Control Interface system was realized by the own developed Brain Visualization and Evaluation Software and VSCP daemon running on the same computer. The Brain Visualization and Evaluation Software acquired and processed the data coming from the EEG device. The attention signal of the EEG device was applied and sent to the controlled node, which was a Control Signal Visualizer test application. It was a simple data comparator and was run on a different computer. The computers were connected to TCP/IP network. If the attention value was higher than the comparator's level, the red light turned on, otherwise it remained turned off. The comparator level can be adjusted by a track bar.

The control application was tested with continuously measured and processed EEG signal. Users can switch on the light if they concentrate strongly according to the level of the comparator adjusted by the track bar. The test environment and test application is shown in Figure 5 and 6.

The effectiveness of the human brain-controlled system operation, which was described above, was examined by Chi-Square Independence Test statistical analysis. The effectiveness of the control was tested at $50 \%$ concentration comparator level while ten test subjects had to switch on the lamp according to a given sequence by varying the concentration intensity during a five minutes test. When the concentration was low the lamp was switched off, and when the concentration was high the lamp was switched on.

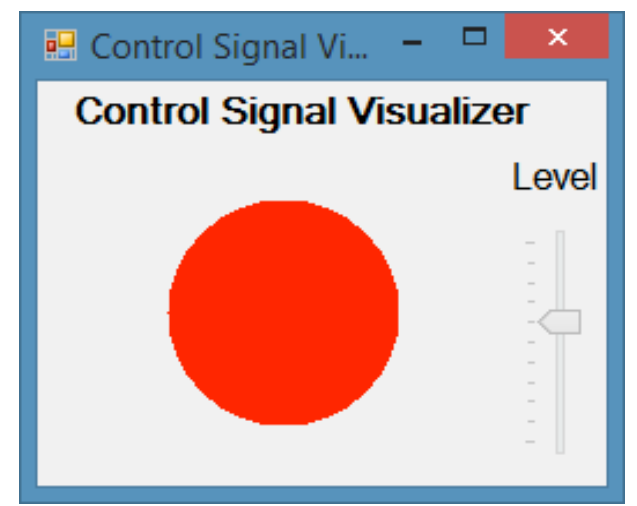

Figure 6. Control Signal Visualizer test application

Data analyses were performed using SPSS statistical software ${ }^{\circledR}$ version 20.0 (SPSS, Inc., Chicago, IL).

TABLE II.

CONTINGENCY TABLE

\begin{tabular}{|c|c|c|c|}
\hline & $\begin{array}{c}\text { brain- } \\
\text { controlled } \\
\text { lamp off }\end{array}$ & $\begin{array}{c}\text { brain- } \\
\text { controlled } \\
\text { lamp on }\end{array}$ & Total \\
\hline $\begin{array}{c}\text { desired } \\
\text { lamp off }\end{array}$ & $\mathbf{1 2}$ & $\mathbf{6}$ & $\mathbf{1 8}$ \\
\hline $\begin{array}{c}\text { desired } \\
\text { lamp on }\end{array}$ & $\mathbf{7}$ & $\mathbf{1 5}$ & $\mathbf{2 2}$ \\
\hline Total & $\mathbf{1 9}$ & $\mathbf{2 1}$ & $\mathbf{4 0}$ \\
\hline
\end{tabular}

Chi square $=4.82$

Asymp. Sig. $=0.028$

Since the P-value is less than the significance level (0.05), we cannot accept the null hypothesis. Thus, we conclude that there is a relationship between desired and brain-controlled lamp status. Regarding the significance test, we report the Pearson Chi-Square value, (degree of freedom $=2$ ) and p-value as in we observed a good association between the current and the preferred brands, $\chi 2(2)=$ $4.82, \mathrm{p}=0.05$. 


\section{CONCLUSION}

The program described above enable users to investigate how brain wave signals - measured by the EEG headset alternate in time and how they depend on the changes of concentration. On the basis of the results, the information received from the processing of brain waves can be used in several research areas; for instance, control, multimedia applications or games etc. The program can easily be further developed and added new functions due to its modular build, which can be the basis of future application developments.

It can be established that good relationship is observable between the sequence of control command series and the commands issued according to concentration level. According to the statistical results the technology does not give perfect results, but it can be assumed on the basis of strong correlation value that the technology will be further developed in the future and even the measurement of brain bioelectric activity become applicable for certain human control tasks.

\section{REFERENCES}

[1] H. Devlin. (2007, Jun 14) What is Functional Magnetic Resonance Imaging (fMRI)? [Online]. Available: http://www. http://psychcentral.com

[2] R. Henson, "Forward inference using functional neuroimaging: dissociations versus associations", Trends in Cognitive Science, vol. 10 , no. 2, pp. 64-69, 2006. http://dx.doi.org/10.1016/j.tics. 2005.12.005

[3] D. C. Knight, C. N. Smith, D. T. Cheng, E. A. Stein, F. J. Helmstetter, "Amygdala and hippocampal activity during acquisition and extinction of human fear conditioning", Cognitive, Affective \& Behavioral Neuroscience, vol. 4, no. 3, pp. 317-325, 2004. http://dx.doi.org/10.3758/CABN.4.3.317

[4] R. Henson, "What can functional neuroimaging tell the experimental psychologist?", The Quarterly Journal of Experimental Psychology, vol. 58a, no. 2, pp. 193-233, 2005. http://dx.doi.org/10.1080/02724980443000502

[5] M. M. Ter-Pogossian, M. E. Phelps, E. J. Hoffman, N. A. Mullani, "A positron-emission transaxial tomograph for nuclear imaging (PET)", Radiology, vol. 114 no. 1, pp. 89-98, 1975 http://dx.doi.org/10.1148/114.1.89

[6] M. E. Phelps, E. J. Hoffman, N. A. Mullani, M. M. Ter-Pogossian, "Application of annihilation coincidence detection to transaxial reconstruction tomography", Journal of Nuclear Medicine, vol. 16, no. 3 , pp. $210-224,1975$.

[7] E. D. Morris, C. C. Constantinescu, J. M. Sullivan, M. D. Normandin, L. A. Christopher, "Noninvasive visualization of human dopamine dynamics from PET images", Neurolmage, vol. 51, no. 1 , pp. 135-144, 2010. http://dx.doi.org/10.1016/j.neuroimage. 2009.12.082

[8] M. S. George, et al. "Noninvasive Techniques for Probing Neurocircuitry and Treating Illness: Vagus Nerve Stimulation (VNS), Transcranial Magnetic Stimulation (TMS) and Transcranial Direct Current Stimulation", Neuropsychopharmacology, vol. 35, no. 1, pp. 301-316, 2010. http://dx.doi.org/10.1038/npp.2009.87

[9] J. J. Taylor, J. J. Borckardt, M. S. George, "Endogenous opioids mediate left dorsolateral prefrontal cortex rTMS-induced analgesia." Pain, vol. 153, no. 6, pp. 1219-1225, 2012 http://dx.doi.org/10.1016/j.pain.2012.02.030

[10] M. C. Ridding, J. C. Rothwell, "Is there a future for therapeuticose of transcranial magnetic stimulation?," Nature Rev. Neurosci, vol 8, no 7, pp. 559-567, Jul, 2007. http://dx.doi.org/10.1038/nrn2169

[11] M. Riehl, "TMS Stimulator Design", in Wassermann, E. M. Epstein, C. Ziemann, U. Walsh, V. Paus, T. Lisanby, Oxford Handbook of Transcranial Stimulation. Oxford: Oxford University Press. pp. 13-23, 2008. http://dx.doi.org/10.1093/oxfordhb/978019 8568926.013.0003

[12] S. Tak, J. Jang, K. Lee, J. C. Ye, "Quantification of CMRO(2) without hypercapnia using simultaneous near-infrared spectroscopy and fMRI measurements.", Phys Med Biol., vol. 55, no. 11, pp 3249-3269, 2010. http://dx.doi.org/10.1088/0031-9155/55/11/017
[13] E. Keller, A. Nadler, H. Alkadhi, S. S. Kollias, Y. Yonekawa, P. Niederer, "Noninvasive measurement of regional cerebral blood flow and regional cerebral blood volume by near-infrared spectroscopy and indocyanine greene dye dilution.", Neuroimage, vol. 20, no. 22, pp. 828-839, 2003. http://dx.doi.org/10.1016/S10538119(03)00315-X

[14] J. Kilner and K. J. Friston, "Topological Inference for EEG and MEG," The Annals of Applied Statistics, vol. 4, no. 3, pp. 11391620, 2010. http://dx.doi.org/10.1214/10-AOAS337

[15] Saeid Sanei and J. A. Chambers, EEG Signal Processing. Cardiff University, UK: John Wiley \& Sons, Ltd, 2007.

[16] NeuroSky Inc, Brain Wave Signal (EEG) of NeuroSky, NeuroSky, 2009.

[17] J. Katona, I. Farkas, T. Ujbanyi, P. Dukan, and A. Kovari, "Evaluation Of The Neurosky MindFlex EEG headset Brain Waves Data," IEEE 12th International Symposium on Applied Machine Intelligence and Informatics, Herl'any, Slovakia, pp. 91-94, 2014. http://dx.doi.org/10.1109/sami.2014.6822382

[18] X. Cui, S. Bray, D. M. Bryant, G. H. Glover, A. L. Reiss, "A quantitative comparison of NIRS and fMRI across multiple cognitive tasks", NeuroImage, vol. 54, no. 4, pp. 2808-2821, 2011 http://dx.doi.org/10.1016/j.neuroimage.2010.10.069

[19] L. T. Vernon, J. Bolaños, D. Suarez, K. Tan, R. Grzeszczuk, Levin, N. David, R. Cakmur, A. F. Samuel, J-P. Spire, "The spatial location of EEG electrodes: Locating the best-fitting sphere relative to cortical anatomy." Electroencephalography and Clinical Neurophysiology, vol. 86, no. 1, pp. 1-6, 1993. http://dx.doi.org/10.1016/0013-4694(93)90061-Y

[20] H. Aurlien, I. O. Gjerde, J. H. Aarseth, G. Eldøen, B. Karlsen, H. Skeidsvoll, N. E. Gilhus, "EEG background activity described by a large computerized database." Clinical Neurophysiology, vol. 115 , no. 3, pp. 665-673, 2004. http://dx.doi.org/10.1016/j.clinph. 2003.10.019

[21] D. O. Creutzfeldt, S. Watanabe, D. H. Lux, "Relations between EEG phenomena and potentials of single cortical cells. I. Evoked responses after thalamic and epicortical stimulation." Electroencephalography and Clinical Neurophysiology, vol. 20, no. 1, pp. 1-18, 1966. http://dx.doi.org/10.1016/0013-4694(66)90136-2

[22] M. E. Whitham, J. K. Pope, S. P. Fitzgibbon, T. W. Lewis, C. C. Richard, S. Loveless, M. Broberg, et al. "Scalp electrical recording during paralysis: Quantitative evidence that EEG frequencies above $20 \mathrm{~Hz}$ are contaminated by EMG." Clinical Neurophysiology, vol. 118 , no. 8, pp. 1877-1888, 2007. http://dx.doi.org/10.1016/j.clinph.2007.04.027

[23] K. Hirai, Wako Res. Center, Honda R\&D Co. Ltd., Saitama, Japan; M. Hirose, Y. Haikawa, T. Takenaka, "The development of Honda humanoid robot," Proceedings Of IEEE International Conference on Robotics and Automation, vol. 2, pp. 1321-1326, 1998.

[24] S. Haufe, M. S. Treder, M. F. Gugler, M. Sagebaum, G. Curio and B. Blankertzm, "EEG potentials predict upcoming emergency brakings during simulated driving." Journal of Neural Engineering, vol. 8, no. 5, 2011. http://dx.doi.org/10.1088/17412560/8/5/056001

[25] K. LaFleur, K. Cassady, A. Doud, K. Shades, E. Rogin and B. He, "Quadcopter control in three-dimensional space using a noninvasive motor imagery-based brain-computer interface," Journal of Neural Engineering, vol. 10, no. 4, 2013. http://dx.doi.org/10.1088/1741-2560/10/4/046003

\section{AUTHORS}

J. Katona, works as a teacher of engineering specialization of IT at University of Dunaújváros. His research areas include engineering, bioinformatics, cognitive psychology and software development. University of Dunaújváros, Dunaújváros, Hungary (e-mail: katonaj@mail.duf.hu).

A. Kovari, works as associate professor at College of Dunaújváros. He received the $\mathrm{PhD}$ degree from the University of Pannonia, Hungary. His main research fields include IT, bioinformatics, control theory and mechatronics. College of Dunaújváros, Dunaújváros, Hungary (email: kovari@mail.duf.hu).

Submitted, 6 October 2015. Published as resubmitted by the authors on 30 October 2015. 